\title{
SEÇÃO DE LEITURA NO TOEFL: UMA ANÁLISE SOB O VIÉS DO LETRAMENTO CRÍTICO
}

\section{Reading Section in TOEFL: an analysis from Critical Literacy perspective}

\author{
Dayse Paulino de ATAIDE, UFPR ${ }^{4}$ \\ Gisele dos Santos da SILVA, UFPR ${ }^{5}$
}

\begin{abstract}
RESUMO: Teste aplicado no mundo inteiro, O TOEFL (Test of English as a Foreign Language - internet based test) já foi realizado por milhões de estudantes e outros indivíduos que pretendem ou precisam comprovar sua proficiência na língua inglesa para atender diversos interesses - acadêmicos, profissionais ou para residir em países onde o inglês é a língua nativa ou a língua requisitada para determinados fins. Nesse trabalho, nosso objetivo é analisar a seção de leitura do TOEFL, a fim de verificarmos se o teste cumpre com algumas premissas relacionadas ao letramento crítico. Escolhemos essa abordagem por acreditarmos que ela vai ao encontro do que o exame preconiza, uma vez que é esperado que os candidatos estejam aptos a interagirem e negociarem sentidos com outros falantes de forma crítica. Para tanto, revisitaremos o conceito de língua de Bakhtin (2014), bem como as teorias de Pennycook e Makoni (2007) e Jordão (2014), que refletem sobre importantes questões linguísticas que nos ajudarão a cumprir o propósito desenhado para este artigo.
\end{abstract}

PALAVRAS-CHAVE: Leitura; Letramento; TOEFL.

ABSTRACT: The TOEFL (Test of English as a Foreign Language - internet based test), a test which is applied in all over the world, has already been done by millions of individuals who aim or need to measure their proficiency in English language in order to answer several purposes - academic, professional or to get a visa to live in a country where English language is the native language or is required to certain goals. In this work we analyze the reading section of TOEFL, since we want to check if the test accomplishes with some assumptions with regard to critical literacy. This theory is used by reason of the exam professes test takers are able to interact and negotiate meanings with other speakers critically. Therefore, we use the Bakhtin's language conception (2014), as well as theories of Pennycook and Makoni (2007) and Jordão (2014), who throw light to important issues which will support us to carry out this paper objectives.

KEYWORDS: Reading; Literacy; TOEFL.

\section{INTRODUÇÃO}

O TOEFL é um dos testes mais realizados por quem deseja avaliar ou comprovar a proficiência em inglês. De acordo com a página eletrônica oficial do exame ${ }^{6}$, diversas agências internacionais, mais de 9000 instituições de ensino e outras entidades de mais de 130 países elegem essa forma de avaliação como critério fundamental para certificar a

\footnotetext{
${ }^{4}$ Mestranda em Letras pela UFPR

${ }^{5}$ Doutoranda em Letras pela UFPR

${ }^{6}$ Disponível em: <https://www.ets.org/Media/Tests/TOEFL/pdf/SampleQuestions.pdf> Acesso em 28/06/2016.
} 
proficiência dos indivíduos que objetivam ingressar em ambientes onde o inglês é requerido para diversos fins, geralmente relacionados com a imersão universitária em uma região onde o inglês é predominante. Destes fatores denota-se a importância em discutirmos algumas implicações do teste no aprendizado e no uso do inglês por falantes de outras línguas ${ }^{7}$.

Neste artigo, partimos desse contexto para pensarmos em uma questão fundamental quando tratamos da língua: o letramento crítico (doravante LC), uma das teorias que abordam o ensino-aprendizagem de língua numa perspectiva sociocultural que julgamos adequada para atender os objetivos deste trabalho. Para tanto, utilizamos um recorte do exame internacional onde esse aspecto pode ser analisado com mais profundidade, a seção de leitura. A fim de nos concentrarmos nesses elementos, este texto foi dividido em duas partes: na primeira, nosso propósito será a descrição da estrutura e objetivos da seção de leitura do TOEFL. Em seguida, estudaremos a seção de leitura sob o viés do LC, pois consideramos relevante confrontar o exame com aquilo que o letramento crítico julga fundamental quando o assunto é língua. Compreendemos que a complexidade do TOEFL permite diferentes análises, mas encontramos no LC um enfoque para o discurso, para a língua construída na coletividade. Sendo assim, considerando que o teste mensura a capacidade do candidato para agir em contextos onde ele se depara com as noções defendidas pelo LC, a abordagem se mostrou coerente. Partindo desta reflexão, buscamos adiante verificar como a seção de leitura do TOEFL atenderia ou não às propostas do LC no que diz respeito à formação do falante que interage criticamente, sabendo lidar com os conflitos e as diferenças que acontecem em sua sociedade. Isso será feito a partir dos apontamentos de Jordão (2014), autora que concebe a leitura como um espaço coletivo onde os sentidos se constroem, em que leitores ajudam a atribuir e não decodificar ou identificar os significados dos textos. Revisitaremos ainda as teorias de Bakhtin (2014), Pennycook e Makoni (2007) e Menezes de Souza (2011), estudiosos que tecem importantes considerações sobre o que devemos ponderar no tratamento da língua em vários contextos, como em relação ao TOEFL, foco do nosso trabalho.

\section{SEÇÃO DE LEITURA DO TOEFL: ESTRUTURA E OBJETIVOS}

Por ser um exame valorizado globalmente tomando como base o número de pessoas que aplicam para o teste, bem como o índice de instituições internacionais que o utilizam

\footnotetext{
${ }^{7}$ Essa pesquisa tem como base uma única amostra do teste, visto que é uma análise inicial sobre a seção de leitura do TOEFL. Ressaltamos o nosso interesse em dar continuidade a esse estudo, aprofundando com a leitura de outras versões da mesma prova.
} 
como requisito parcial em processos seletivos, são importantes recorrentes pesquisas em como o TOELF é construído, em seus objetivos e nas lacunas que podem afetar o rendimento dos candidatos, especialmente no que se refere à leitura, seção selecionada para análise.

De acordo com a página eletrônica oficial do exame, mais de 30 milhões de pessoas que já realizaram o teste o fizeram por diversas razões, a saber:

01. Estudantes que pretendem ingressar em universidades no exterior;

02. Candidatos participantes de processos de admissão em programas universitários;

03. Estudantes universitários que aplicam para bolsas de estudos no exterior;

04. Aprendizes de língua que desejam acompanhar o próprio processo de aquisição da língua inglesa;

05. Trabalhadores e estudantes de precisem de visto (ETS, 2016).

Os dados obtidos no meio eletrônico do próprio exame indicam que o TOEFL tem um papel bastante contundente nas universidades e outras instituições ao redor do mundo, especialmente nos Estados Unidos e Canadá. Como uma ilustração, podemos citar o FLTA (Foreign Language Teaching Assistant), da Comissão Fulbright Brasil, programa cujo interesse é atrair graduados brasileiros que desejam ensinar português nos Estados Unidos, mas que possuam proficiência em inglês comprovada por um dos seguintes testes: TOEFL ou IELTS (FULBRIGHT, 2015).

Em relação à amostra analisada neste artigo, utilizamos um exemplo disponibilizado no site do TOEFL em $2015^{8}$ (a seção de leitura está em Anexo). De acordo com a descrição da página, o texto, bem como as questões, aos quais tivemos livre acesso foram utilizados em testes anteriores, algo que consideramos bastante significativo, uma vez que julgamos relevante refletir sobre um teste que já foi aplicado previamente.

Com respeito ao conteúdo, a passagem liberada para exame intitula-se "Meteorite Impact and Dinosaur Extinction", um texto de 57 linhas acompanhado por 14 questões objetivas, que serão discutidas no decorrer do artigo. De acordo com as orientações cedidas aos candidatos e aos interessados em realizar ou conhecer a estrutura da prova,

A seção de leitura mensura sua habilidade para entender passagens acadêmicas escritas em inglês. Você lerá uma passagem e responderá questões sobre ela. No

\footnotetext{
${ }^{8}$ No site do TOEFL iBT é possível acessarmos apenas um dos três ou quatro textos aplicados em ocasiões anteriores. O tempo total reservado para realização da leitura e resolução das questões correspondente à passagem selecionada corresponde a 20 minutos. No teste completo, os candidatos têm entre 60-80 minutos para responderem de 36-56 questões.
} 
teste verdadeiro do TOEFL iBT, você teria 20 minutos para ler a passagem e responder as questões. Candidatos com deficiências podem requerer um tempo maior. (ETS, 2015) ${ }^{9}$

Na próxima seção, nos dedicaremos à análise da parte de leitura do TOEFL em relação a alguns pressupostos do LC, pois apreendemos que há uma grande distância entre o que o teste defende como necessário para medir a proficiência linguística dos candidatos e o que ele deveria considerar para demonstrar que os candidatos estão aptos para usar o inglês nos contextos sociais em que a língua circula.

\section{A LEITURA NO TOEFL E O LETRAMENTO CRÍTICO}

Tendo em vista o ensino de língua estrangeira, o progresso esperado do aprendiz e a concepção do LC, buscamos verificar se a seção de leitura do TOEFL atende às proposições do LC no que diz respeito ao processo de aprendizagem da língua e o desenvolvimento do falante. Justificamos a nossa abordagem teórica pelos próprios objetivos do exame, que calcula o quanto o examinando é capaz de usar a língua no nível universitário, de acordo com o órgão que gerencia e aplica o TOEFL. Nesse sentido, o teste não está desvinculado do ensino do idioma avaliado, ou seja, pressupomos que ele pode interferir nos modos como o inglês é ensinado e estudado por quem se dispõe a realizar a prova. Fica a dúvida, inclusive, se o indivíduo não busca os cursos de inglês para se preparar para o TOEFL, tornando-o compatível com algumas premissas do LC.

Segundo o LC (JORDÃO, 2014; MENEZES DE SOUZA, 2011), a língua é entendida como discurso e este, por sua vez, possibilita a construção de sentidos. Tal visão é corroborada pelo pensamento bakhtiniano o qual postula que a língua é um construto social e, portanto, traz em si uma carga socioideológica do contexto de uso. Dessa maneira, a língua permite que haja significação na interação verbal em diferentes contextos sociais. Ainda nessa perspectiva, o ensino de língua estrangeira possibilita o contato com diferentes discursos e a significação de diversas situações em que a interação acontece por meio do idioma inglês. Sendo assim, a sala de aula dessa disciplina pode ser concebida como o espaço onde professores e alunos podem atribuir sentidos ao mundo a partir de suas experiências e realidades. Como afirmam Jordão e Fogaça (2007, p. 93), "ao questionar pressupostos e implicações de diferentes pontos de vista na sociedade - e nos textos - e assim ampliar

\footnotetext{
${ }^{9}$ No original: The Reading section measures your ability to understand academic passages written in English. You will read one passage and answer questions about it. In the actual TOEFL iBT® test, you would have 20 minutes to read the passage and answer the questions. Test takers with disabilities can request a time extension.
} 
nossas perspectivas, é possível que os alunos sejam capazes de também perceber-se enquanto sujeitos críticos, capazes de agir sobre o mundo e seus sentidos.”. Em outras palavras, o LC no ensino da língua inglesa possibilita que o aluno questione, reflita e ressignifique os diferentes textos e discursos que circulam socialmente, e desenvolva uma posição crítica perante a sociedade, compreendendo-se como um agente em seu meio social.

Posto isso, o nosso aporte teórico prevê que a produção de significado é situacional e tem o leitor como peça fundamental nesse processo. Sendo assim, espera-se que cada indivíduo seja um leitor crítico dos textos que o rodeia. Para isso, Menezes de Souza (2011, p.132) afirma que

Ler criticamente implica, então, em desempenhar pelo menos dois atos simultâneos e inseparáveis: (1) perceber não apenas como o autor produziu determinados significados que tem origem em seu contexto e seu pertencimento sócio-histórico, mas ao mesmo tempo, (2) perceber como, enquanto leitores, a nossa percepção desses significados e de seu contexto sócio-histórico está inseparável de nosso próprio contexto sócio-histórico e os significados que dele adquirimos. (Grifos no original)

Dito de outra maneira, ser um leitor crítico é perceber-se como sujeito também responsável pela concepção dos sentidos dos textos, sendo capaz de compreender que os significados não são previamente definidos, mas são negociados no momento da leitura, podendo haver diversas significações para um mesmo texto.

No decorrer da nossa análise, constatamos em primeira instância que, no TOEFL, ainda presenciamos a falta do espaço para que os sentidos sejam construídos, como preconiza o LC. Nesse exame, é exigido que os candidatos busquem informações que se encontram na superfície dos textos, sem que se estabeleça o diálogo entre a passagem e os leitores. Em resumo, os textos são acadêmicos, mas de áreas muito peculiares, ou seja, que não atendem ou se articulam com as outras áreas de conhecimento. A nossa amostra, por exemplo, traz uma parte de um artigo científico que trata do impacto dos meteoritos na extinção dos dinossauros e em outros aspectos da vida no nosso planeta. Além disso, é um texto da geologia que pode causar dúvidas naqueles candidatos oriundos de campos do conhecimento muito distintos e que podem não compreender com facilidade as particularidades do texto, como o vocabulário. Apesar de as questões não exigirem um conhecimento lexical e temático complexo, consideramos que um teste tão importante a nível mundial deveria contemplar assuntos de ampla relevância social que abrangesse várias áreas, países e contextos. Em outras palavras, constatamos pela passagem e questões que o exame está distante da concepção de língua como prática social, pois não dá abertura para que os candidatos 
construam os significados para o que ele está lendo (BAKHTIN, 2014). Conforme advoga Jordão,

No letramento crítico, então, a leitura e o desenvolvimento da criticidade vão além do material, do verbal, dos sentidos supostamente "dados" nos textos, para a exploração do processo de atribuição de sentidos aos textos, processo entendido como não linear e não apenas racional. Além das condições de produção dos textos, importam no letramento crítico as condições de produção das leituras, bem como suas implicações para a vida dos sujeitos leitores (JORDÃO, 2014, p.139).

Quando confrontamos a citação acima com o teste em análise, nos deparamos com algumas contradições, a começar pelas questões que requerem dos candidatos sinônimos que tenham um significado próximo de algumas palavras ou sentenças específicas do texto base. Em nosso exemplo, isso pode ser notado especialmente nas questões 1, 5, 6, 8 e 9. Nelas, os examinados podem fazer uma leitura linear do texto a fim de escolherem a alternativa mais adequada conforme solicitado no enunciado da questão. Como uma ilustração, observe a questão abaixo:

\section{The word "pose" on line 2 is closest in meaning to}
a) claim
b) model
c) assume
d) present

(ETS, 2015, p.2)

Nela, é solicitado uma palavra que tenha um significado similar à pose, expressão que pode ser lida na segunda linha da passagem, sem ser necessário, assim, ter conhecimento da totalidade do texto para respondê-la. Em relação às outras perguntas, salientamos que a maioria apresenta possibilidades limitadas de interpretação, ou seja, não deixa evidente que a alternativa considerada correta é apenas uma entre outras opções de leitura. As questões 2, 4, 7, 10,11, 13 e 14 explicitam essa característica do exame. Nelas podemos perceber algumas habilidades recorrentes, tais como: reconstituição da argumentação (questão 2 e 11), localização pontual e linear da informação (questões 4, 7 e 12), reconhecimento do quadro enunciativo (questão 10), bem como ordenação e relevância (questões 13 e 14). Ademais, pelo menos oito delas se aproximam de alguns aspectos aos quais Nery (2003, p.100) nomeou como "reconstituição de informações dispersas ao longo do texto" e "seleção e ordenação de informações segundo seu grau de relevância”. No exame, isso é requerido nas perguntas 2, 3, $4,7,10,11,12$ e 14, em que os examinados precisam identificar informações pontuais a partir de partes específicas do texto. 
Apesar de considerarmos essas habilidades fundamentais para o desenvolvimento do leitor, o trabalho com elas não precisa ser feito de maneira mecanizada, em que o candidato deva se concentrar meramente em buscar a resposta adequada, como acontece no TOEFL.

Nesse cenário, destacamos ainda a abordagem da inferência na questão 3, uma das capacidades leitoras mais importantes na construção de significados do texto. Segundo Koch e Elias (2006), é necessário considerar os conhecimentos prévios do leitor e sua condição para se estabelecer a interação entre os textos e leitores. Assim, por mais que o teste trate de competências linguísticas elementares para certificar leitores (NERY, 2003) sua abordagem se afasta do que defende o LC, uma vez que o teste acaba estabelecendo limites aos seus candidatos.

Nesse caso, ousamos dizer que esse teste pode estar distante de provar que os candidatos estão aptos ou não para usar a língua inglesa. É perfeitamente possível que os indivíduos consigam usar a língua em diversos contextos, mas no teste eles precisam se deparar com outras demandas que vão além do linguístico, como o tempo que eles têm para realização da prova (para a passagem seria reservado 20 minutos para a leitura e resolução de questões), a não familiaridade com os temas dos textos, a tensão do momento, as condições dos locais onde as provas são realizadas, entre outras que variam em cada ambiente. De acordo com Makoni e Pennycook (2007), a língua é uma invenção do homem, resultante dos movimentos sociais e culturais de um povo. Eles ainda advogam que ela não resiste ao tempo e recebe a interferência de vários fatores que afetam a sua existência, o que acarreta na sua desiventação, assim como sua reinvenção . O TOEFL, tratado como uma invenção linguística, foi criado a partir de uma pequena parcela da língua por homens que desconsideram as peculiaridades dos indivíduos que o realizam, mas não participam da sua criação. Nesse sentido, concordamos com os autores quando afirmam que

\begin{abstract}
nós deveríamos discutir os efeitos materiais muito reais das invenções linguísticas, uma vez que elas influenciam como as línguas têm sido entendidas, como as políticas linguísticas têm sido construídas, como a educação tem sido buscada, como os testes de língua têm sido desenvolvidos e administrados, e como pessoas passaram a identificar com rótulos particulares e às vezes até morrer por eles, como a natureza violenta de rivalidade étnica na África, Ásia do Sul e qualquer lugar amplamente demonstra. Dessa forma, enquanto as entidades em torno das batalhas são travadas, os testes que são construídos e as políticas linguísticas que são escritas são invenções, os efeitos são muito verdadeiros (MAKONI e PENNYCOOK, 2007, p. $2-3)^{10}$.
\end{abstract}

\footnotetext{
${ }^{10}$ No original: "we would argue for the very real material effects of linguistic inventions since they influence how languages have been understood, how language policies have been constructed, how education has been pursued, how language tests have been developed and administered, and how people have come to identify with particular labels and at times even to die for them, as the violent nature of ethnic rivalry in Africa, South Asia
} 
Além disso, esse tipo de questão, que espera que o sujeito seja capaz de estabelecer relações entre léxico e texto, dificilmente proporciona aos candidatos um espaço de conflitos ou negociação de sentidos, permitindo-lhes que se faça uma leitura crítica do texto, uma vez que exige apenas a assimilação de palavras ao contexto dado, contrário ao que o próprio teste diz fazer, avaliar a língua em uso.

Similarmente, questões que se configuram conforme os exercícios 4, 7 e 12 do teste aqui analisado, os quais solicitam que o candidato encontre uma informação pontualmente localizada no texto, não propiciam o uso da língua para construção de sentidos em tal contexto. De fato, mesmo não havendo a compreensão global do texto, uma pessoa que não seja necessariamente proficiente na língua inglesa também seria capaz de identificar a resposta da pergunta presente no texto, uma vez que esta requer uma informação pontual dentro do quadro enunciativo do texto. Desse modo, pouco espaço é destinado para a reflexão e a transformação advindas da ação interpretativa do texto, como prevê o LC quanto ao uso da língua, por exemplo.

Nesse sentido, o teste caminha na direção oposta da concepção de língua

como um espaço de construção coletiva de sentidos, envolvendo uma história (ou várias) social, cultural, política, econômica, uma história do passado, do presente e do futuro, entendida numa perspectiva bakhtiniana como "arena de conflitos" e "língua do outro" (Bakhtin, 1999), como espaço onde sentidos se constroem [...] (JORDÃO, 2014, p. 135).

Pode-se afirmar que o TOEFL contraria os objetivos e as necessidades que levam os candidatos a realizarem uma prova de proficiência. Conforme abordado na outra seção, os indivíduos que realizam o teste geralmente intencionam ingressar em uma instituição de ensino, morar em um país estrangeiro, entre outros motivos. Dessa forma, seria necessário que o exame levasse em consideração que os examinandos utilizam o inglês em contextos que exigem criticidade e a interação com outros falantes para que juntos negociem decisões diante das demandas da sociedade. Em outras palavras, cidadãos que tenham "a percepção crítica de estar com o mundo vem através da conscientização social e crítica de que nunca estamos sozinhos no mundo" (MENEZES DE SOUZA, 2011, p.130).

Além disso, se pensarmos que para haver uma percepção crítica no uso da língua é necessário considerar o contexto do falante para a negociação de sentidos, quando o candidato se depara com um teste como o TOEFL, criado por uma instituição norteamericana que tem como escopo a cultura norte-americana, o candidato poderá encontrar

and elsewhere amply demonstrates. Thus, while the entities around which battles are fought, tests are constructed and language policies are written are inventions, the effects are very real. 
algumas dificuldades, uma vez que alguns temas podem não corresponder com a realidade local do indivíduo. Provavelmente, se o teste fosse organizado conforme a realidade de cada país que o utiliza, trazendo textos diversificados e com temas que considerassem os contextos locais dos indivíduos, as particularidades da vida acadêmica de cada cultura, os candidatos poderiam desfrutar de uma situação que atendesse seus horizontes de expectativas, permitindo assim uma negociação crítica de sentidos mais profícua.

Questionamento semelhante a este é apresentado por Brock-Utne (2012) em seu artigo Language and inequality: global challenges to education em que a autora aborda os desafios da internacionalização e o ensino na África, destacando como o regime de aplicação de testes internacionais atingem o continente. Neste texto, Brock-Utne (2012) relata a tentativa de pesquisadores da área da educação de desvencilhar o ensino africano de currículos, materiais didáticos, testes e todo tipo de ideia ligados ao modelo adotado por países colonizadores, buscando uma educação de qualidade pautada em seus próprios contextos. Isso posto, ela traz o exemplo do uso de um teste internacional (o TIMSS - Trend in International Mathematics and Science Studies) em Gana e o baixo desempenho dos alunos neste teste, uma vez que além de ser aplicado na língua não nativa (no caso, o teste é feito em inglês), ele se situa em um contexto ocidental, utilizando conceitos culturais e linguísticos com os quais os alunos ganeses não estavam familiarizados, prejudicando-os em seus resultados. Em outras palavras, este exemplo reforça que para haver a negociação de sentido é de considerável importância que o exame seja condizente com o contexto sociocultural do candidato.

A mesma situação exposta anteriormente pode acontecer com candidatos ao realizarem o TOEFL. Se considerarmos o modelo do teste aqui analisado e a aplicação deste no Brasil para falantes brasileiros estudantes de inglês como segunda língua, por exemplo, certamente muitos candidatos teriam dificuldades em respondê-lo, não apenas pela pouca reflexão exigida na resolução das questões, mas principalmente pelo conteúdo e o contexto textual que são delimitados conforme o país de origem (EUA), que muitas vezes pode ser compreendido com mais facilidade pelos falantes nativos. Dessa forma, o indivíduo avaliado, além de não alcançar um bom resultado no exame, também não terá a possibilidade de fazer uma leitura crítica em situações que se distanciem muito de sua realidade. Portanto, repensar o exame de acordo com o LC também levantaria a discussão em torno dessa questão da descontextualização de temas e textos segundo cada local em que este é aplicado.

Outra questão que nos chamou a atenção diz respeito ao formato do teste, isto é, ao fato de este ser dividido em seções conforme a habilidade a ser explorada em cada uma delas. 
Tal divisão remete ao modelo adotado na perspectiva da abordagem comunicativa, sendo esta a abordagem mais utilizada nos países em que o inglês é a língua oficial, justificando assim o porquê de o TOEFL apresentar o trabalho com as habilidades em seções diferentes (McKAY, 2003). Dessa maneira, o exame traz além da seção de compreensão escrita aqui analisada, as seções de compreensão oral, produção oral e escrita. Como o nome indica, a abordagem comunicativa de ensino de língua tem como objetivo o estabelecimento da comunicação utilizando a língua alvo; propõe-se também o uso de textos autênticos e acredita-se que os modelos comunicativos de uso da língua aprendidos em simulações em sala de aula são representações da interação que acontece no mundo real, e o conhecimento dessas habilidades desenvolvidas em sala possibilita que o falante seja capaz de se comunicar em qualquer situação por meio da língua alvo (McKAY, 2003).

No entanto, se consideramos o LC uma abordagem que melhor se adequa à formação do falante crítico de uma língua estrangeira, para que haja a negociação de sentidos não basta apenas o uso de textos autênticos, é necessário que o texto e o processo de ensinoaprendizagem sejam vistos como um todo, onde as quatro habilidades são trabalhadas de forma híbrida. Em outras palavras, separar a avaliação das habilidades em diferentes seções acarreta o entendimento de que, nas situações corriqueiras, utilizamos uma habilidade dissociada da outra, como se não fossem fatores complementares na interação verbal. Como aponta McKay (2003), não é só o modelo do falante nativo que interfere no ensinoaprendizagem do inglês em diversos países, mas o método de ensino adotado, assim como o formato de teste de proficiência empregado, também advém da cultura de países em que o inglês é a língua oficial, reforçando o imperialismo linguístico que atinge muitas nações não falantes da língua inglesa. Por conseguinte, McKay (2003) ainda ressalta que não existe uma pedagogia ou um método específico para o ensino de inglês no cenário internacional, já que, segundo a autora, uma metodologia adequada para o ensino de inglês deve ser sensível o bastante para levar em conta a cultura de cada local e de cada contexto de sala de aula em que o processo de aprendizagem acontece, considerando os anseios, conflitos e dificuldades aparentes em cada situação de interação na língua.

Portanto, novamente enfatizamos que a formatação do TOEFL poderia ser revisada trabalhando com os textos em uma perspectiva diferente sem que haja a divisão das habilidades em si, visando dessa forma criar situações em que o candidato possa realmente fazer uma leitura crítica dos textos e negociar sentidos de acordo com a sua realidade, sentindo-se agente ao utilizar a língua inglesa, seja durante o teste, seja nas interações diversas de uso do idioma. O LC não é especificamente uma metodologia que indica passo a 
passo como se deve ensinar ou aprender uma língua, porém é uma abordagem que ressalta a necessidade de o processo de ensino-aprendizagem proporcionar ao sujeito a percepção de que os sentidos são negociados no uso da língua em cada situação interacional diferente e, acima de tudo, notar que cada falante precisa se posicionar crítica e reflexivamente nessas interações, reconhecendo que o seu ponto de vista também é válido em relação ao ponto de vista de seu interlocutor; que não existem verdades absolutas, mas sim perspectivas adotadas por cada indivíduo que precisam ser compreendidas, respeitadas e principalmente negociadas.

\section{CONSIDERAÇÕES FINAIS}

Ainda que o TOEFL tenha ampla aplicação internacionalmente como um teste de proficiência em língua inglesa e considerando as seções que o compõem, ele não atende ao que se espera em consonância a uma abordagem crítica de uso da língua. Quando falamos em criticidade, partimos da perspectiva do LC, que entende a língua como fator social que permite a negociação de sentido na formação dos discursos que nos envolvem a cada situação comunicacional. Dessa maneira, a língua é discurso e por meio dele criamos sentido para as coisas de nosso mundo. Nesse caso, os textos permitem a constante negociação de sentidos, ressignificando-nos. Porém, o exame aqui analisado não parece promover situações em que seja possível haver a negociação e a ressignificação por parte do candidato que o presta.

Além da fragmentação do trabalho com as habilidades, constatamos que o TOEFL costuma ser composto por questões extremamente lineares, pontuais, que exigem apenas a localização da informação ou o simples emprego da sinonímia dentro do texto. Esses tipos de questões não requerem do candidato uma leitura crítica e nem aceita outras possibilidades de respostas em relação ao texto, limitando a construções de sentidos perante o mesmo. Além disso, a escolha de temas não condizentes com os horizontes de expectativas dos candidatos pode acarretar dificuldades na compreensão do texto e, consequentemente, baixos resultados naquilo que corresponderia à proficiência na língua inglesa. Novamente, acreditamos que para haver de fato a negociação de sentidos e o desenvolvimento da criticidade do candidato, além da utilização de textos autênticos, os temas, gêneros e vocabulários utilizados deveriam ser condizentes com o contexto sociocultural de quem o realiza, e não ser configurado da mesma forma para todas as nações levando em conta o modelo adotado por um país oficialmente anglofalante. Concomitantemente, é preciso repensar se um teste baseado na abordagem comunicativa seria profícuo em nosso contexto sociocultural, por exemplo, se este realmente comprovaria a proficiência de um indivíduo no momento de interação em 
situações reais de uso da língua e se a fragmentação das habilidades assemelhar-se-ia às situações do mundo real.

Em suma, seria interessante que o TOEFL se adequasse mais aos contextos diversos em que é aplicado, correspondendo às expectativas dos candidatos de cenários socioculturais e historicamente diferentes, deixando de ser formatado conforme a cultura e a metodologia seguida por países anglófonos, aqueles que são vistos como modelos a serem alcançados e donos da língua alvo em si. Além disso, seria necessário que houvesse maior flexibilidade para as respostas e maior espaço para negociação de sentidos durante o teste, para que o candidato fosse capaz de se perceber enquanto sujeito agente na constituição e compreensão dos diferentes discursos encontrados. Certamente o TOEFL ainda será um teste de referência em muitas situações e em diversos países. No entanto, não precisamos simplesmente aceitá-lo como o mais adequado em nosso contexto, sem questioná-lo ou refletir sobre suas contribuições e implicações na formação dos sujeitos críticos. Não problematizar o teste significa desconsiderar as teorias recentes sobre ensino-aprendizagem da língua inglesa, bem como os contextos de uso de seus falantes, não apenas brasileiros, mas de países do mundo inteiro.

\section{REFERÊNCIAS}

BAKHTIN, M. (VOLOSHINOV). Marxismo e filosofia da linguagem. 16. ed. São Paulo: Editora Hucitec, 2014.

BROCK-UTNE, B. Language and inequality: global challenges to education. In: Compare: A Journal of Comparative and International Education. Vol: 42, 773-793, 2012.

EDUCATIONAL SERVICE. About the TOEFL iBT Test. Disponível em $<$ https://www.ets.org/toefl/ibt/about $>$ Acesso em 28/06/2016.

FULBRIGHT Brasil. FLTA: Programa para ensinar português nos EUA. Disponível em $<$ http://fulbright.org.br/edital/programa-para-ensinar-portugues-nos-eua-flta/ $>$ Acesso em 07/07/2016.

JORDÃO, C. Aprendendo língua estrangeira com o professor Jacotot: criticidade na pedagogia crítica e no letramento crítico. In: MATEUS, E; OLIVEIRA, N. Estudos críticos da linguagem e formação de professores de línguas: contribuições teórico-metodológicas. Campinas: Pontes, 2014.

JORDÃO, C; FOGAÇA, F.C. Ensino de Inglês, Letramento Crítico e Cidadania: um Triângulo Amoroso Bem-Sucedido. In: Línguas e Letras. 2007 Vol 8, n.14, p. 79-105.

KOCH, I. V.; ELIAS, V. M. Ler e compreender os sentidos do texto. São Paulo: Contexto, 2006.

MAKONI, S; PENNYCOOK, A. Desinventing and Reconstituting Languages. Clevedon, UK: Multilingual Matters, 2007.

MCKAY, S.L. Toward an appropriate EIL pedagogy: re-examining common ELT assumptions. International Journal of Applied Linguistics, v. 13, n.1, 2003. p. 1-22.

MENEZES DE SOUZA, L. M. T. Para uma redefinição de Letramento Crítico: conflito e produção de Significação. In: MACIEL, Ruberval Franco; ARAUJO, Vanessa de Assis 
(Orgs.) Formação de professores de línguas: ampliando perspectivas. Jundiaí: Paco editorial, 2011.

NERY, R. M. Ler em língua materna, ler em língua estrangeira: a questão é de leitura. In: Norma Sandra de Almeida Ferreira. (Org.). Um cons/certo - 25 anos COLE. São Paulo: Companhia Ed. Nacional \& ABL, 2003, p. 97-114.

\section{ANEXO: SEÇÃO DE LEITURA}

\section{Meteorite Impact and Dinosaur Extinction}

There is increasing evidence that the impacts of meteorites have had important effects on Earth, particularly in the field of biological evolution. Such impacts continue to pose a natural hazard to life on Earth. Twice in the twentieth century, large meteorite objects are known to have collided with Earth.

If an impact is large enough, it can disturb the environment of the entire Earth and cause an ecological catastrophe. The best-documented such impact took place 65 million years ago at the end of the Cretaceous period of geological history. This break in Earth's history is marked by a mass extinction, when as many as half the species on the planet 10 became extinct. While there are a dozen or more mass extinctions in the geological record, the Cretaceous mass extinction has always intrigued paleontologists because it marks the end of the age of the dinosaurs. For tens of millions of years, those great creatures had flourished. Then, suddenly, they disappeared.

The body that impacted Earth at the end of the Cretaceous period was a meteorite with a mass of more than a trillion tons and a diameter of at least 10 kilometers. Scientists first identified this impact in 1980 from the worldwide layer of sediment deposited from the dust cloud that enveloped the planet after the impact. This sediment layer is enriched in the rare metal iridium and other elements that are relatively abundant in a meteorite but 20 very rare in the crust of Earth. Even diluted by the terrestrial material excavated from the crater, this component of meteorites is easily identified. By 1990 geologists had located the impact site itself in the Yucatán region of Mexico. The crater, now deeply buried in sediment, was originally about 200 kilometers in diameter.

This impact released an enormous amount of energy, excavating a crater about twice as large as the lunar crater Tycho. The explosion lifted about 100 trillion tons of dust into the atmosphere, as can be determined by measuring the thickness of the sediment layer formed when this dust settled to the surface. Such a quantity of material would have blocked the sunlight completely from reaching the surface, plunging Earth into a period 30 of cold and darkness that lasted at least several months. The explosion is also calculated to have produced vast quantities of nitric acid and melted rock that sprayed out over much of Earth, starting widespread fires that must have consumed most terrestrial forests and grassland. Presumably, those environmental disasters could have been responsible for the mass extinction, including the death of the dinosaurs.

Several other mass extinctions in the geological record have been tentatively identified with large impacts, but none is so dramatic as the Cretaceous event. But even without such specific documentation, it is clear that impacts of this size do occur and that their results can be catastrophic. What is a catastrophe for one group of living things, however, 40 may create opportunities for another group. Following each mass extinction, 
there is a sudden evolutionary burst as new species develop to fill the ecological niches opened by the event.

Impacts by meteorites represent one mechanism that could cause global catastrophes and 45 seriously influence the evolution of life all over the planet. According to some estimates, the majority of all extinctions of species may be due to such impacts. Such a perspective fundamentally changes our view of biological evolution. The standard criterion for the survival of a species is its success in competing with other species and adapting to slowly changing environments. Yet an equally important criterion is the ability of a species to 50 survive random global ecological catastrophes due to impacts.

Earth is a target in a cosmic shooting gallery, subject to random violent events that were unsuspected a few decades ago. In 1991 the United States Congress asked NASA to investigate the hazard posed today by large impacts on Earth. The group conducting the 55 study concluded from a detailed analysis that impacts from meteorites can indeed be hazardous. Although there is always some risk that a large impact could occur, careful study shows that this risk is quite small.

1. The word "pose" on line 2 is closest in meaning to
a. claim
b. model
c. assume
d. present

2. In paragraph 2, why does the author include the information that dinosaurs had flourished for tens of millions of years and then suddenly disappeared?

a. To support the claim that the mass extinction at the end of the Cretaceous is the best-documented of the dozen or so mass extinctions in the geological record

b. To explain why as many as half of the species on Earth at the time are believed to have become extinct at the end of the Cretaceous

c. To explain why paleontologists have always been intrigued by the mass extinction at the end of the Cretaceous

d. To provide evidence that an impact can be large enough to disturb the environment of the entire planet and cause an ecological disaster

3. Which of the following can be inferred from paragraph 3 about the location of the meteorite impact in Mexico?

a. The location of the impact site in Mexico was kept secret by geologists from 1980 to 1990 .

b. It was a well-known fact that the impact had occurred in the Yucatán region.

c. Geologists knew that there had been an impact before they knew where it had occurred.

d. The Yucatán region was chosen by geologists as the most probable impact site because of its climate.

4. According to paragraph 3, how did scientists determine that a large meteorite had impacted Earth?

a. They discovered a large crater in the Yucatán region of Mexico.

b. They found a unique layer of sediment worldwide.

c. They were alerted by archaeologists who had been excavating in the Yucatán region.

d. They located a meteorite with a mass of over a trillion tons.

5. The word "excavating" on line 25 is closest in meaning to

a. digging out 

b. extending
c. destroying
d. covering up

6. The word "consumed" on line 32 is closest in meaning to
a. changed
b. exposed
c. destroyed
d. covered

7. According to paragraph 4, all of the following statements are true of the impact at the end of the Cretaceous period EXCEPT:
a. A large amount of dust blocked sunlight from Earth.
b. Earth became cold and dark for several months.
c. New elements were formed in Earth's crust.
d. Large quantities of nitric acid were produced.

8. The phrase "tentatively identified" on line 36 is closest in meaning to
a. identified after careful study
b. identified without certainty
c. occasionally identified
d. easily identified

9. The word "perspective" on line 46 is closest in meaning to
a. sense of values
b. point of view
c. calculation
d. complication

10. Paragraph 6 supports which of the following statements about the factors that are essential for the survival of a species?

a. The most important factor for the survival of a species is its ability to compete and adapt to gradual changes in its environment.

b. The ability of a species to compete and adapt to a gradually changing environment is not the only ability that is essential for survival.

c. Since most extinctions of species are due to major meteorite impacts, the ability to survive such impacts is the most important factor for the survival of a species.

d. The factors that are most important for the survival of a species vary significantly from one species to another.

11. Which of the sentences below best expresses the essential information in the following sentence?

Earth is a target in a cosmic shooting gallery, subject to random violent events that were unsuspected a few decades ago.

Incorrect choices change the meaning in important ways or leave out essential information.

a. Until recently, nobody realized that Earth is exposed to unpredictable violent impacts from space.

b. In the last few decades, the risk of a random violent impact from space has increased.

c. Since most violent events on Earth occur randomly, nobody can predict when or where they will happen.

d. A few decades ago, Earth became the target of random violent events originating in outer space. 
12. According to the passage, who conducted investigations about the current dangers posed by large meteorite impacts on Earth?
a. Paleontologists
b. Geologists
c. The United States Congress
d. NASA

13. Look at the four letters $(\mathrm{A}, \mathrm{B}, \mathrm{C}$, and D) that indicate where the following sentence could be added to the passage in paragraph 6 .

\section{This is the criterion emphasized by Darwin's theory of evolution by natural selection.}

Where would the sentence best fit?

Impacts by meteorites represent one mechanism that could cause global catastrophes and seriously influence the evolution of life all over the planet. (A) According to some estimates, the majority of all extinctions of species may be due to such impacts. (B) Such a perspective fundamentally changes our view of biological evolution. (C) The standard criterion for the survival of a species is its success in competing with other species and adapting to slowly changing environments. (D) Yet an equally important criterion is the ability of a species to survive random global ecological catastrophes due to impacts.

Choose the place where the sentence fits best.
a. Option A
b. Option B
c. Option C
d. Option D

14. An introductory sentence for a brief summary of the passage is provided below. Complete the summary by selecting the THREE (3) answer choices that express the most important ideas in the passage. Some sentences do not belong in the summary because they express ideas that are not presented in the passage or are minor ideas in the passage. This question is worth 2 points.

Write your answer choices in the spaces where they belong. You can write in the number of the answer choice or the whole sentence.

\section{Scientists have linked the mass extinction at the end of the Cretaceous with a meteorite impact on Earth.}

\section{Answer choices}

(1) Scientists had believed for centuries that meteorite activity influenced evolution on Earth.

(2) The site of the large meteorite impact at the end of the Cretaceous period was identified in 1990.

(3) There have also been large meteorite impacts on the surface of the Moon, leaving craters like Tycho.

(4) An iridium-enriched sediment layer and a large impact crater in the Yucatán provide evidence that a large meteorite struck Earth about 65 million years ago.

(5) Large meteorite impacts, such as one at the end of the Cretaceous period, can seriously affect climate, ecological niches, plants, and animals.

(6) Meteorite impacts can be advantageous for some species, which thrive, and disastrous for other species, which become extinct. 